Original Research

\title{
The Effect of Curing Temperature on the Morphology and Electro-Optical Properties of a Flexible UV-Cured Polymer Dispersed Liquid Crystal (PDLC)
}

\author{
Hassan-Ali Hakemi * \\ Plastic Liquid Crystal Technology, Via Lambro 80, 20846 Macherio (MB), Italy; E-Mail: \\ hassanalihakemi@gmail.com
}

* Correspondence: Hassan-Ali Hakemi; E-Mail: hassanalihakemi@gmail.com

Academic Editor: Hossein Hosseinkhani

Special Issue: Polymer Dispersed Liquid Crystal Technology

Recent Progress in Materials

2020 , volume 2 , issue 4

doi:10.21926/rpm.2004023
Received: October 11, 2020

Accepted: November 22, 2020

Published: December 8, 2020

\begin{abstract}
In this work, the experimental data on flexible PDLC films at the industrial scale and the effect of cure temperature $\left(T_{c}\right)$ on the morphology and electro-optical properties of a UV-cured PDLC system via polymer-induced phase separation (PIPS) method were studied. Under constant UV radiation intebsity and thickness, the morphological parameters such as dimension, number density, and volume fraction of the phase-separated liquid crystal droplets as well as the optical transmissions and switching voltages as a function of cure temperature were determined. The effect of cure temperature on the morphology and electro-optical properties of UV-cured PDLC films were analyzed.
\end{abstract}

\section{Keywords}

UV-cured flexible PDLC; cure temperature; morphology; optical transmissions; switching voltages

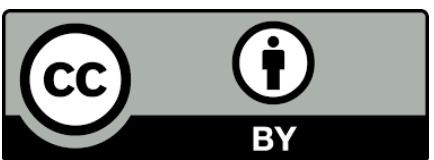

(C) 2020 by the author. This is an open access article distributed under the conditions of the Creative Commons by Attribution License, which permits unrestricted use, distribution, and reproduction in any medium or format, provided the original work is correctly cited. 


\section{Introduction}

The field of Polymer Dispersed Liquid Crystal (PDLC) has been the subject of intense academic and industrial research and development in the past four decades. The study of the correlation between PDLC materials and process conditions on the morphology and electro-optical properties is essential for industrial development and manufacture. The chemical structure and physical properties of raw materials, the phase separation process, and curing of the matrix are important parameters that influence the morphology and electro-optical properties of PDLC films. The literature mostly deals with academic studies on the effect of material and process conditions, such as type, composition, temperature, radiation, time, and thickness, on the electro-optical properties of various types of PDLC films.

The recent studies on thermoset PDLC films have shown that the morphology and electro-optical properties of the PDLC devices are affected by the type of material and process parameters, including the concentration, cure temperature, cure time, and thickness [1-9]. In this respect, the optimal inter-dependent material and process parameters need to be identified with experimental verification of their correlations. The electro-optical properties of the thermoset PDLC film depend on several factors, such as the type of liquid crystal, the molecular structure of pre-polymers, and polymeric conditions. These factors are found to affect the microstructure of the polymer matrix, the size and shape of the LC domains, and the anchoring of the LC molecules within the droplets [10-13].

In this work, the effect of cure temperature $\left(T_{c}\right)$ on the morphology and electro-optical properties of a flexible UV-cured PDLC system was studied by the polymer-induced phase separation (PIPS) method. This study was part of an industrial R\&D program to establish the empirical relationship between the curing parameters and morphology, and also between the morphology and electrooptics for the subsequent scale-up and manufacture of PDLC products. At the industrial scale, these relationships will serve to develop quantitative correlations between the process parameters and electro-optical properties of PDLC films and are indispensable for the optimization of product quality during the manufacturing process.

\section{Experimental}

\subsection{Materials and Preparation}

The utilized materials were as follows: UV-curable NOA65 pre-polymer resin (Norland Optical Adhesives), TNO623 nematic mixture (Hoffmann-La Roche), $15 \mathrm{~m}$ plastic micro-spacer Micropearl (Sekisui), photoinitiator Irgacure819 (Sigma-Aldrich), and $100 \Omega$ resistor. The ITO-PET film supports were received from Sheldahl. All materials were used without further purification. The thermal and optical properties of the TNO623 liquid crystal mixtures, such as nematic-isotropic transition temperature $\left(T_{n i}\right)$, birefringence $(\Delta n)$, ordinary $\left(n_{0}\right)$ and extraordinary $\left(n_{e}\right)$ refractive indices, resistivity $(\rho)$, dielectric anisotropy $(\Delta \varepsilon)$, and viscosity $(\eta)$, are summarized in Table I.

Table 1 Thermal and optical properties of TNO623 liquid crystal mixture.

\begin{tabular}{cc}
\hline Property & TNO623 \\
\hline $\mathrm{T}_{\text {ni }}\left({ }^{\circ} \mathrm{C}\right)$ & 105
\end{tabular}




$\begin{array}{cc}\Delta \mathrm{n} & 0.198 \\ \mathrm{n}_{\mathrm{o}} & 1.507 \\ \mathrm{n}_{\mathrm{e}} & 1.705 \\ \rho\left(10^{8} \Omega . \mathrm{m}\right) & 10.5 \\ \Delta \varepsilon & 17.2 \\ \eta(\mathrm{mPa}) & 66\end{array}$

The PDLC was prepared via the polymerization-induced phase separation (PIPS) method by UV irradiation (UV-PIPS). The flexible PDLC film was prepared from the custom-made in-situ coating and lamination system $[8,9]$ of a homogeneous mixture of NOA65 pre-polymer $(50 \%)$, TNO623 (50\%), Irgacure 819 (1\% vs. total formulation), and plastic micro-spacers ( $0.2 \%$ vs. total formulation) in weight percentage, which was pre-heated at $50{ }^{\circ} \mathrm{C}$ for $10 \mathrm{~min}$. The uncured PDLC mixture was then poured into the vertical gap between the two rolls of ITO-PET support films. The uncured PDLC film immediately under the coating rolls was passed through a pressure roll to ensure the uniformity of the film by micro-spacers. The uncured PDLC film sandwich was cut and transferred to a custombuilt conveyor. This was further cured using a high-intensity UV lamp of $50 \mathrm{~mW} / \mathrm{cm}^{2}$ radiation intensity at a line speed of 0.5 meters/minute under the relevant cure temperatures of $25,45,60$, and $85^{\circ} \mathrm{C}$. The cure temperature was measured using a thermocouple during the curing process. The experiments were performed on four PDLC samples for each formulation, and the reported experimental results were the average of four samples.

\subsection{Methods and Measurements}

The electro-optical properties of the PDLC films, such as off-state transmission ( $\left.\mathrm{T}_{\text {off }}\right)$, on-state transmission $\left(\mathrm{T}_{\mathrm{o}}\right)$, threshold voltage $\left(\mathrm{V}_{10}\right)$, and saturation voltage $\left(\mathrm{V}_{90}\right)$, were measured using a specially constructed photometric system consisting of a white light source, sample chamber, photometer, amplifier, function generator, and electronic data acquisition network as reported previously $[8,9]$.

The morphological parameters of the PDLC films, including the average micro-droplet dimension $(\langle D\rangle)$, number density per unit area $(\langle N\rangle)$, and droplet volume fraction $(\langle\alpha\rangle)$, were measured from the areas of the horizontal and fractured micrographs using the JOEL model JSM-6300 scanning electron microscope (SEM). The values of $\langle\mathrm{D}\rangle$ and $\langle\mathrm{N}\rangle$ were determined by the direct twodimensional image analysis of the SEM micrographs of the samples. The PDLC layer thicknesses were also measured from the SEM micrographs and Mitutoyo-7007 specimeter. The resistivity $\rho$ of the TN0623 liquid crystal was measured at $1 \mathrm{~V}$ and $100 \mathrm{~Hz}$ frequency, and its nematic-isotropic transition temperature was measured using the Nikon Eclipse-50i polarizing optical microscope equipped with LTS120 Linkam hot-stage.

\section{Results \& Discussion}

\subsection{Effect of Curing Temperature on Morphology}

The experimental results of the effect of curing temperature $\left(T_{c}\right)$ on the droplet morphology of the UV-cured PDLC films containing the TNO623 mixture are presented. The morphological 
parameters such as the average liquid crystal droplet diameter $(\langle\mathrm{D}\rangle)$, number density $(\langle\mathrm{N}\rangle)$, and volume fraction $(\langle\alpha\rangle)$ were measured from the SEM micrographs of $15 \mu \mathrm{m}$ thick samples. These morphological parameters are tabulated as a function of the four cure temperatures $(25,45,60$, and $85{ }^{\circ} \mathrm{C}$ ) at constant cure UV radiation intensity of luv $=50 \mathrm{~mW} . \mathrm{cm}^{2}$ and line speed of 0.5 meters/minute in Table 2.

Table 2 The effect of cure temperature on the morphology of TNO623-PDLC.

\begin{tabular}{ccccc}
\hline PDLC & $\mathrm{T}_{\mathrm{c}}\left({ }^{\circ} \mathrm{C}\right)$ & $\langle\mathrm{D}\rangle(\boldsymbol{\mu \mathrm { m }})^{\mathrm{b}}$ & $\langle\mathrm{N}\rangle\left(\mathbf{1 0 ^ { 5 }} \cdot \mathrm{cm}^{-\mathbf{2}}\right)^{\mathrm{c}}$ & $\langle\boldsymbol{\alpha}\rangle^{\mathrm{d}}$ \\
\hline UV25 & 25 & 3.2 & 16.5 & 0.15 \\
UV45 & 45 & 2.5 & 33.0 & 0.18 \\
UV60 & 60 & 1.6 & 83 & 0.19 \\
UV85 & 85 & 1.35 & 135 & 0.22 \\
\hline
\end{tabular}

In general, the volume fraction of the phase-separated liquid crystal droplets $(\langle\alpha\rangle)$ is inversely proportional to the amount of liquid crystal plasticized in the matrix. The $\langle\alpha\rangle$ values of the TNO623PDLC system were calculated from the morphological data using the following equation:

$$
<\alpha>=\pi \cdot X /(100-X) \cdot\left(\rho_{\mathrm{re}} / \rho_{\mathrm{lc}}\right) \cdot<\mathrm{N}>\cdot(<\mathrm{D}>/ 2)^{2}
$$

where $\langle\mathrm{N}\rangle / \mathrm{A}$ and $\langle\mathrm{D}\rangle$ are as described above, $\mathrm{X}$ is the utilized liquid crystal weight percentage $(50 \%)$, and $\rho_{\mathrm{re}}=1.15$ and $\rho_{\mathrm{lc}}=1.03$ are the average specific densities of TNO623 and cured NOA65, respectively. The results of the calculated $\langle\alpha\rangle$ values from Equation (1) are also presented in Table 2. As expected, there is a systematic decrease in $\langle\mathrm{D}\rangle$ and increase in $\langle\mathrm{N}\rangle$ with the increase in the temperature within the PDLC system. From Table 2, the morphologies of the TNO623-PDLC films were plotted as a function of cure temperature, as shown in Figure 1. Under the studied UV intensity and temperature range, the morphologies of the present TNO623-PDLC films exhibited the expected decrease in $\langle D\rangle$ and increase in $\langle N\rangle$, but an unexpected increase in $\langle\alpha\rangle$ with the temperature. The unexpected behavior of $\langle\alpha\rangle$ may be attributed to the process condition of the PDLC film with a confined thickness of $15 \mathrm{~mm}$, resulting in rather large droplet dimensions. The relatively small $<\alpha>$ values indicate that more than $80 \%$ of TNO623 has been plasticized in the matrix due to the present process conditions.
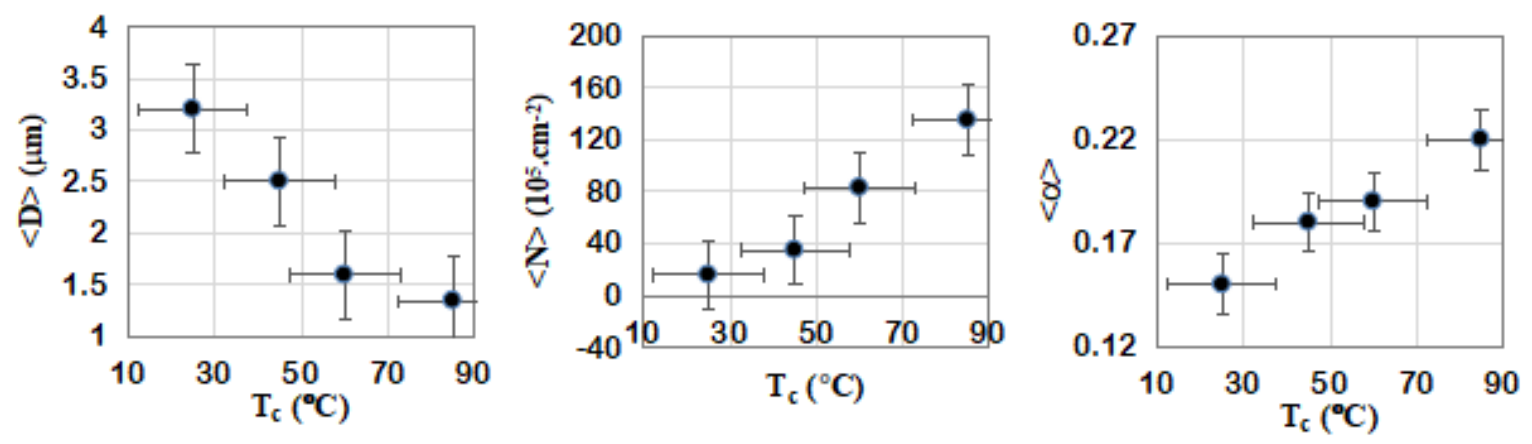

Figure 1 The morphological parameters of TNO623-PDLC as a function of cure temperature. 
The inverse temperature dependency of $\langle D\rangle$ is consistent with the previously reported literature data in UV-curable and thermoset PDLC systems [14-23].

The effect of temperature on morphology explains that the rate of phase separation is larger than the matrix polymerization rate, leading to a decrease in $\langle\mathrm{D}\rangle$ but an increase in the $\langle\mathrm{N}\rangle$ and $\langle\alpha\rangle$ values with cure temperature. In other words, the matrix polymerization kinetics is dominant, and the increase in the solubility of TNO623 and pre-polymer results in high matrix plasticization. This explains the decrease in $\langle D\rangle$ but not the increase in $\langle N\rangle$ and $\langle\alpha\rangle$ with increasing cure temperature in the TNO623-PDLC system, where the phase separation occurs primarily near the coexistence curve that results in a favorable increase in the rate of matrix polymerization against phase separation, leading to favorable morphology and electro-optical properties. It should be noted that the inverse trend in the $\angle D>$ values with cure temperature is consistent with the literature data on the PDLC films developed using UV-curable PIPS and solvent-induced phase separation (SIPS) methods [14-16] presented by the following empirical relationship in Equation (2) [17].

$$
<\mathrm{D}>=\mathrm{A} \cdot \mathrm{e}^{-\left(\mathrm{B} \cdot \mathrm{T}_{\mathrm{C}}\right)}
$$

where $A$ and $B$ are the experimental parameters of the system. The nonlinearity of $\angle D>v s T_{c}$ is typical of the PDLC system. According to Equation (2), a semi-logarithmic plot of $\angle D>v s 1 / T_{c}$ was constructed that displayed a linear trend, as presented in Figure 2. This is in agreement with the other thermoset PDLC systems that show diverging or converging trends depending on the thermoset formulation $[17,18]$.

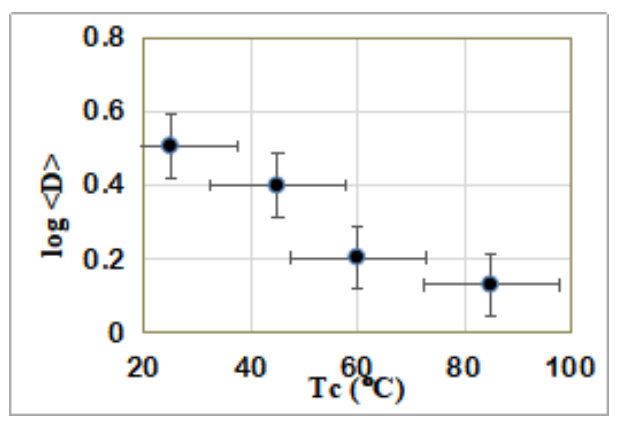

Figure 2 The plot of log $<D>$ versus $T_{c}$ in TNO623-PDLC based on Equation (2).

From the experimental data in Table 2, the relationship between $\langle\mathrm{D}\rangle$ and $\langle\mathrm{N}\rangle$ of the phaseseparated LC droplets was deduced as a function of $T_{c}$. The droplet number densities $\langle N\rangle$ in the PDLC system increased nonlinearly with cure temperature, as observed in thermoset PDLC films [23]. This effect is attributed to the increase in solubility and decrease in viscosity of the formulation materials that in turn results in a decrease in phase separation, increase in the matrix curing rate, and subsequently, the creation of smaller droplets and larger matrix plasticization at higher temperatures.

\subsection{Effect of Curing Temperature on the Electro-optical Properties}

The electro-optical properties of the TN0623-PDLC films were studied within the cure temperature range of $25-85^{\circ} \mathrm{C}$. The optical transmissions ( $\left.T_{\text {on }} \& T_{\text {off }}\right)$ and switching voltages $\left(V_{10} \&\right.$ $\mathrm{V}_{90}$ ) given in Table 3 are plotted, as shown in Figure 2. 
Table 3 The Electro-optics of TNO623-PDLC films as a function of curing temperature.

\begin{tabular}{cccccc}
\hline PDLC & $\mathrm{T}_{\mathbf{c}}\left({ }^{\circ} \mathrm{C}\right)$ & $\mathrm{T}_{\text {on }}(\%)$ & $\mathrm{T}_{\text {off }}(\%)$ & $\mathrm{V}_{\text {10 }}$ (volt) & $\mathrm{V}_{\mathbf{9 0}}$ (volt) \\
\hline UV25 & 25 & 56.4 & 11.0 & 14 & 53 \\
UV45 & 45 & 45.2 & 6.0 & 16 & 65 \\
UV60 & 60 & 44.7 & 2.7 & 18 & 72 \\
UV85 & 85 & 48.8 & 4.0 & 22 & 98 \\
\hline
\end{tabular}
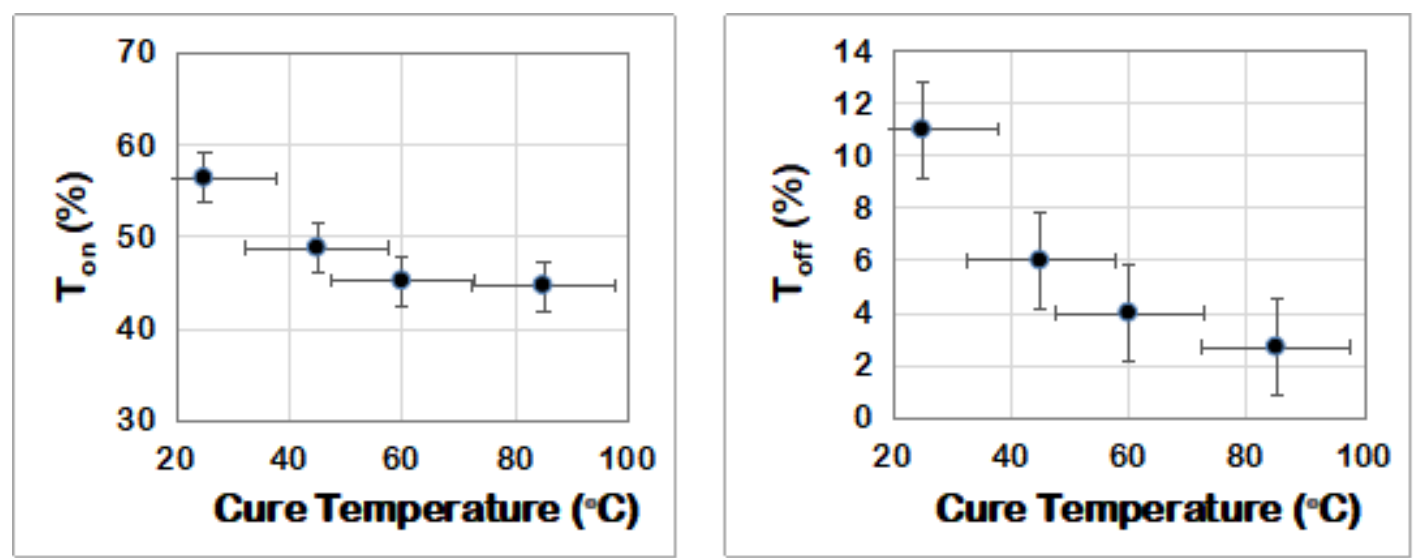

Figure 3 The optical transmissions $T_{\text {on }}$ (left) and $T_{\text {off }}$ (right) of TNO623-PDLC as a function of cure temperature.

From the data in Table 3, the optical transmissions of the TNO623-PDLC films were plotted as a function of cure temperature, as shown in Figure 3. The $T_{\text {on }}$ and $T_{\text {off }}$ values exhibit decreasing trends with temperature, as shown in Figure 3 . It should be noted that the relatively small $T_{\text {on }}$ and large $T_{\text {off }}$ values in the PDLC system under the corresponding process conditions result from the relatively large values of $\langle D\rangle$, the large difference in the refractive indices of NOA65 $\left(n_{p}=1.524\right)$ and TNO623 $\left(n_{0}=1.507\right)$, as well as the influence of high-intensity UV radiation on the confined geometry of 15 $\mathrm{mm}$ thick PDLC layer. These effects may contribute to the decreasing trend in $\mathrm{T}_{\text {off }}$ with temperature, which is in contrast to the thermoset PDLC studies [23]. At low morphology, the light scatters less, leading to large $\mathrm{T}_{\text {off }}$ (opacity) and small $\mathrm{T}_{\text {on }}$ (transmittance) values. There is an exponential relationship between optical transmission, number density, thickness, and scattering cross-section that can be expressed by equation (3) [24].

$$
\mathrm{T}=\mathrm{T}^{0} \cdot \mathrm{e}^{-(<\mathrm{N}>\cdot \mathrm{d} \cdot \sigma)}
$$

where $T$ is the transmitted light intensity, $T^{0}$ is the incident light intensity, $\langle N\rangle$ is the unitary droplet number density, $d$ is the thickness of the PDLC layer, and $\sigma$ is the total scattering crosssection as the droplet scattering cross-section is averaged over the whole region of the PDLC. Using the data in Table 3, a plot of $\mathrm{T}_{\text {off }}$ and $\mathrm{T}_{\text {on }}$ versus $<\mathrm{N}>$ of the TNO623-PDLC films was drawn, as shown in Figure 4. The plot was consistent with Equation (3) and confirmed the exponential divergences of the optical transmissions with droplet number density $\langle\mathrm{N}\rangle$. 

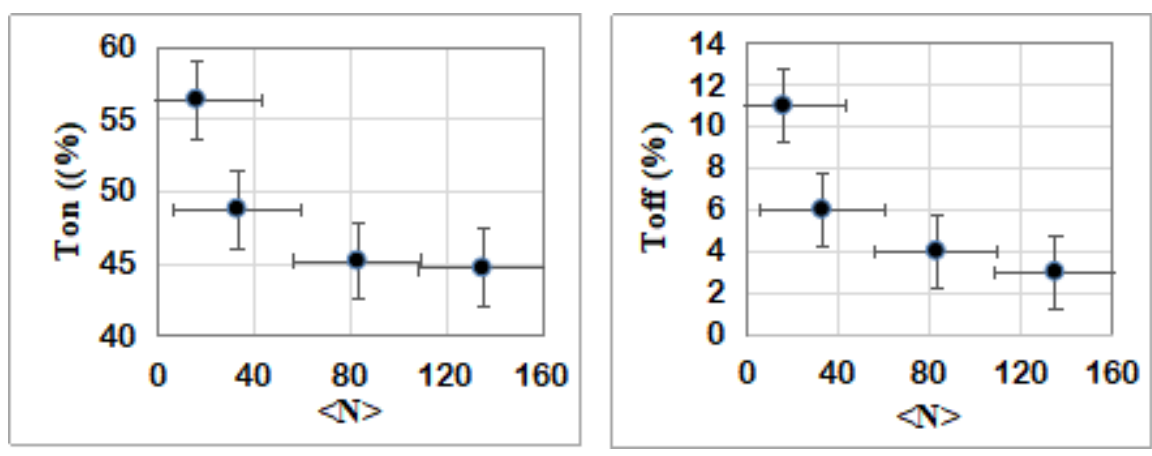

Figure 4 The optical transmissions $\mathrm{T}_{\text {on }}$ (left) and $\mathrm{T}_{\text {off }}$ (right) of TNO623-PDLC versus $<\mathrm{N}>$ according to Equation (3).

Using the data in Table 3, a graph was plotted for the operating voltages of the TNO623-PDLC films as a function of cure temperature, as shown in Figure 5. The results indicate that the threshold voltage $\left(\mathrm{V}_{10}\right)$ and saturation voltage $\left(\mathrm{V}_{90}\right)$ increase linearly with cure temperature. Under constant $U V$ radiation, the dependencies of $V_{10}$ and $V_{90}$ on $T_{c}$ are mainly attributed to the difference in the droplet size and anchoring energy. Besides, the droplet anchoring energy prohibits the alignment of liquid crystals with the electric field by decreasing the value of $<D>$ with temperature (see Table 2), resulting in higher switching voltages.

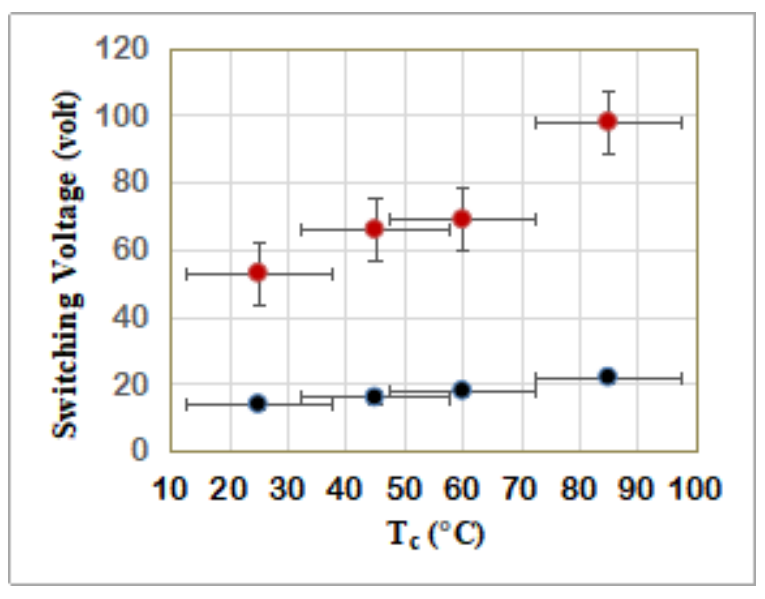

Figure 5 The $V_{10}$ (black) and $V_{90}$ (red) of TNO623-PDLC films as a function of cure temperature.

The increasing gap between $V_{10}$ and $V_{90}$ with cure temperature in Figure 5 is another indication of the contributions of the droplet $\langle\mathrm{D}>$ value and anchoring energy. The same trends were reported for the dependencies of $V_{10}$ and $V_{90}$ on the cure temperature in other thermoset and UV-curable PDLC films [14, 25]. A similar relationship was observed between the morphology and switching voltages, attributing to the effect of the thickness in UV-curable and thermoset PDLC films $[8,9]$. Based on the threshold voltage of PDLC, the correlation of $V_{10}$ with droplet radius $\langle D / 2\rangle$ can be presented by Equation (4) [25].

$$
\mathrm{V}_{10}=\mathrm{d} /<\mathrm{D} / 2>\cdot\left(\rho_{\mathrm{p}}+2 / \rho_{\mathrm{lc}}\right) \cdot\left\{(\mathrm{K}-1) /\left(\varepsilon_{11} \cdot \Delta \varepsilon\right)\right\}^{1 / 2}
$$


where $d$ is the film thickness, $\angle D / 2>$ is the droplet radius, $\rho_{\mathrm{p}}$ and $\rho_{\mathrm{lc}}$ are the respective resistivity of the matrix and $L C, K$ is the average elastic constant, and $\varepsilon_{11}$ and $\Delta \varepsilon$ are the parallel and anisotropy of the dielectric constant, respectively. Equation (4) shows that $V_{10}$ is inversely proportional to $\langle\mathrm{D} / 2\rangle$, as shown in Figure 6 . From the limited details in this study, the apparent nonlinearity of $V_{10}$ and $<\mathrm{D} / 2>$ may be attributed to the nonlinear dependencies of resistivity, and elastic and dielectric parameters with cure temperature.

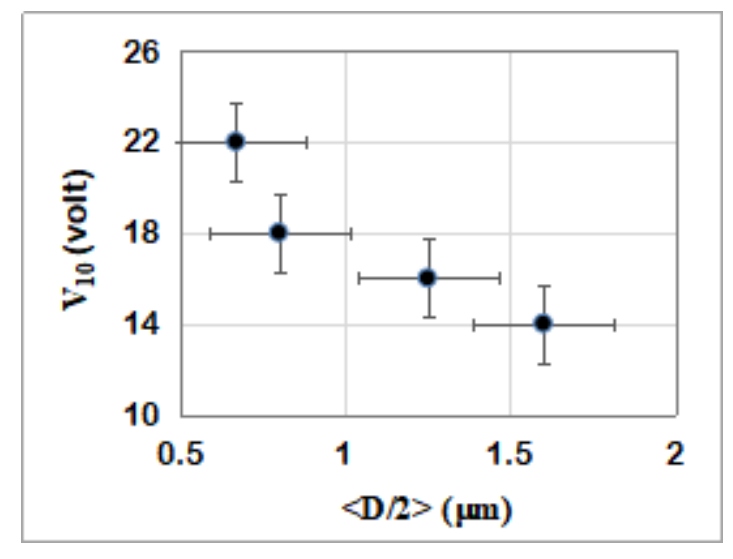

Figure 6 The plot of $V_{10}$ versus $<D / 2>$ in TNO623-PDLC films.

\section{Conclusions}

This study showed the dependency of the morphology of a flexible UV-cured TNO623-PDLC system on the cure temperature under constant intensity UV radiation. The effect of cure temperature on the morphology and electro-optical properties of PDLC, as well as the correlations between the morphological parameters, namely, $\langle\mathrm{D}\rangle,\langle\mathrm{N}\rangle$ and $\langle\alpha\rangle$, were found to be consistent with the existing literature on thermoset and UV-curable PDLC systems. The morphology of the PDLC films exhibited the expected trends in $\langle D\rangle$ and $\langle N\rangle$, but an unexpected trend in $\langle\alpha\rangle$ with temperature under the UV intensity and temperature range used in this study.

The effects of cure temperature on the optical transmissions and operating voltages of the TN0623-PDLC films were studied. The experimental results indicated that the PDLC films exhibited a decrease in the optical transmissions but an increase in the operating voltage values with cure temperature due to the influence of solubility, index mismatch, phase separation, and matrix curing kinetics. As a result, the cure temperature dependency of the electro-optical properties of the TN0623-PDLC films is directly correlated with the morphology, resulting from the phase separation and polymer curing mechanisms under the corresponding process conditions and confined geometry. Under low cure temperatures (near the coexistence curve) in the UV-cured PDLC system, the polymerization rate was lowered, and the phase separation was enhanced, resulting in larger morphologies and poor electro-optical properties. However, at higher cure temperatures (far from the coexistence curve), the extent of polymerization overcomes and suppresses the phase separation mechanism, resulting in smaller droplet size and higher switching properties. Obviously, the cure temperature at the coexistence curve of the polymerization kinetics energetically favors the phase separation mechanism. The ideal industrial-scale materials and process conditions for the formation of UV-cured PDLC were found to be the temperature near the coexistence curve that 
defines the optimum formulation, composition, and cure conditions for any polymer and liquid crystal system.

\section{Acknowledgments}

The experimental part of this work had been carried out at Specialty Film Division of Snia Research, Snia BPD (FIAT Group), Pisticci Scalo (MT) Italy, under Italian funded research project 1.1.4 "Messa a punto di film con proprietà elettro-ottiche" during 1993-1999 period.

\section{Author Contributions}

Hassan-Ali Hakemi did all the research work of this study.

\section{Competing Interests}

The authors have declared that no competing interests exist.

\section{References}

1. Bouteiller L, Barny PL. Polymer-dispersed liquid crystals: Preparation, operation and application. Liq Cryst. 1996; 21: 157-174.

2. Cheng SX, Bai RK, Zou YF, Pan CY. Electro-optical properties of polymer dispersed liquid crystal materials. J Appl Phys. 1996; 80: 1991-1995.

3. Hoppe CE, Galante MJ, Oyanguren PA, Williams RJ. Polymer-dispersed liquid crystals with cocontinuous structures generated by polymerization-induced phase separation of EBBA-epoxy solutions. Macromolecules. 2002; 35: 6324-6331.

4. Hoppe CE, Galante MJ, Oyanguren PA, Williams RJ. Optical properties of novel thermally switched PDLC films composed of a liquid crystal distributed in a thermoplastic/thermoset polymer blend. Mater Sci Eng C. 2004; 24: 591-594.

5. Yamaguchi R, Sakurai S. Cell thickness dependence on electric optical property of reverse mode liquid crystal display. J Photopolym Sci Technol. 2014; 27: 287-290.

6. An YJ, Guo XL, Zhang SH, Du ZQ. Preparation and performance testing of flexible PDLC films. Adv Mater Res. 2014; 1015: 89.

7. Ellahi M, Rafique MY. Electro-optical properties of PDLC films using diethylenetriamine (DETA) hardener. Mol Cryst Liq Cryst. 2016; 638: 103-110.

8. Hakemi $\mathrm{H}$. The effect of thickness on morphology and electro-optics of plastic thermoset polymer dispersed liquid crystal (PDLC). Mol Cryst Liq Cryst. 2019; 681: 12-22.

9. Hakemi H. The effect of thickness on electro-optical properties of plastic UV-curable polymer dispersed liquid crystal (PDLC). Mol Cryst Liq Cryst. 2019; 689: 34-43.

10. Li W, Cao Y, Kashima M, Kong L, Yang H. Effects of the structures of polymerizable monomers on the electro-optical properties of UV cured polymer dispersed liquid crystal films. J Polym Sci B: Polym Phys. 2008; 1: 1369-1375.

11. Ha KR, Ahn W. Temperature effects on LC droplets formation of pdlc films with thermoplastic matrix. Korea Polym J. 1999; 7: 130-135. 
12. He J, Bin Y, Wang X, Yu B, Wang Y. A novel polymer dispersed liquid crystal film prepared by reversible addition fragmentation chain transfer polymerization. Eur Polym J. 2007; 43: 40374042.

13. Ahmad F, Jamil M, Jeon YJ, Woo LJ, Jung JE, Jang JE, Lee GH, Park JJ. Comparative study on the electrooptical properties of polymer-dispersed liquid crystal films with different mixtures of monomers and liquid crystals. J Appl Polym Sci. 2011; 121: 1424-1430.

14. Carter SA, LeGrange JD, White W, Boo J, Wiltzius P. Dependence of the morphology of polymer dispersed liquid crystals on the UV polymerization process. J Appl Phys. 1997; 81: 5992.

15. Folkes RL. Controlling the morphological and electro-optical properties of polymer dispersed liquid crystals. Durham: Durham University; 2008.

16. Challa SR, Wang SQ, Koenig JL. Thermal induced phase separation of $E_{7} / P M M A$ PDLC system. J Thermal Analysis. 1995; 45: 1297-1312.

17. Montgomery GP, West JL, Tamura-Lis W. Light scattering from polymer-dispersed liquid crystal films: Droplet size effects. J Appl Phys. 1991; 69: 1605.

18. West JL. Phase separation of liquid crystals in polymers. Mol Cryst Liq Cryst Incorporating Nonlinear Opt. 1988; 157.

19. Smith GW. Study of formation, phase behavior, and microdroplet size of a polyurethane-based polymer-dispersed liquid crystal. Mol Cryst Liq Cryst. 1990; 180: 201-222.

20. Smith GW. Cure parameters and phase behavior of an ultraviolet-cured polymer-dispersed liquid crystal. Mol Cryst Liq Cryst. 1991; 196: 89-102.

21. Vas NA, Smith GW, Montgomery GP. A light control film composed of liquid crystal droplets dispersed in an epoxy matrix. Mol Cryst Liq Cryst. 1987; 146: 17-34.

22. Smith GW, Vas NA. The relationship between formation kinetics and microdroplet size of epoxybased polymer-dispersed liquid crystals. Liq Cryst. 1988; 3: 543-571.

23. Hakemi $\mathrm{H}$. The effect of curing temperature and time on morphology and Electro-Optical properties of flexible thermoset polymer dispersed liquid crystal (PDLC) films. Mol Cryst Liq Cryst. 2020; 703: 1-12.

24. Akins RB, West JL. Effect of thickness on PDLC electro-optics. Proc SPIE. 1992; 1665: 280-289.

25. Ellahi M, Gao Y, Rafique MY. Influence of enhanced curing temperature of epoxy monomers structure on the electro-optical properties and morphology of polymer-dispersed liquid crystal films. Am J Eng. Res. 2013; 2: 1-6. 


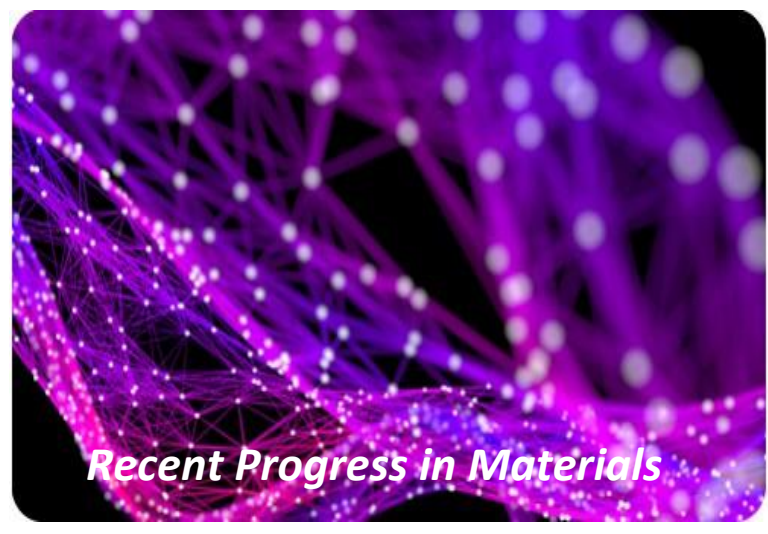

Enjoy Recent Progress in Materials by:

1. Submitting a manuscript

2. Joining in volunteer reviewer bank

3. Joining Editorial Board

4. Guest editing a special issue

For more details, please visit:

http://www.lidsen.com/journals/rpm 